\title{
The Multi-Interval-Valued Fuzzy Soft Set with Application in Decision Making
}

\author{
Shawkat Alkhazaleh \\ Department of Mathematics, Faculty of Sciences and Art, Shaqra University, Shaqra, Saudi Arabia \\ Email:shmk79@gmail.com
}

Received 25 May 2015; accepted 12 July 2015; published 16 July 2015

Copyright (C) 2015 by author and Scientific Research Publishing Inc.

This work is licensed under the Creative Commons Attribution International License (CC BY).

http://creativecommons.org/licenses/by/4.0/

c) (i) Open Access

\begin{abstract}
In 1999, Molodtsov introduced the concept of soft set theory as a general mathematical tool for dealing with uncertainty. By combining the multi-fuzzy set and soft set models, Y. Yang, X. Tan and C. Meng introduced the concept of multi-fuzzy soft sets and studied some of its operations, such as complement, “AND”, “OR", Union and Intersection. They also gave an algorithm to analyze a decision problem using multi-fuzzy soft set. In this paper, we introduce the concept of multi-intervalvalued fuzzy soft set (M-IVFSS). We also define its basic operations, namely complement, union, intersection, AND and OR. Finally, we give an application of this concept in decision-making problem.
\end{abstract}

\section{Keywords}

\section{Soft Set, Fuzzy Soft Set, Multi-Fuzzy Soft Set, Multi-Interval-Valued Fuzzy Soft Set}

\section{Introduction}

Most of the problems in engineering, medical science, economics, environments etc. have various uncertainties. Molodtsov [1] initiated the concept of soft set theory as a mathematical tool for dealing with uncertainties. Chen et al. [2] and Maji et al. in [3] and [4] studied some different operations and application of soft sets. Furthermore, Maji et al. [5] presented the definition of fuzzy soft set and Roy et al. [6] presented the applications of this notion to decision making problems. By using fuzzy sets, Ahmad and Kharal in 2009 [7] studied this theory and defined arbitrary fuzzy soft union and fuzzy soft intersection and proved DeMorgan's Inclusions and DeMorgan's Laws in fuzzy soft set theory. In 2010, Feng et al. [8] gave deeper insights into decision making based on fuzzy soft sets. They discussed the validity of the Roy-Maji method and showed its true limitations. By means of level soft sets, they presented an adjustable approach to fuzzy soft set based decision making and gave some illustrative examples. Moreover, the weighted fuzzy soft set is introduced and its application to decision making is also 
investigated.

The concept of soft fuzzy set and some properties of soft fuzzy set are discussed in 2008 by Yao et al. [9] and the relations of fuzzy soft sets and soft fuzzy sets are compared by instances. Kharal and Ahmad in 2009 [10] defined the concept of a mapping on classes of fuzzy soft sets and studied the properties of fuzzy soft images and fuzzy soft inverse images of fuzzy soft sets, and supported them with examples and counterexamples.

Chaudhuri and K. De in 2009 [11] defined the concepts of soft relation and fuzzy soft relation and applied them to solve a number of decision making problems. The advantages of fuzzy soft relation compared to other paradigms are discussed. Jiang et al. [12] presented in 2011 an extended fuzzy soft set theory by using the concepts of fuzzy description logics to act as the parameters of fuzzy soft sets. They also defined some operations for the extended fuzzy soft sets. Moreover, they proved that certain DeMorgan's laws hold in the extended fuzzy soft set theory with respect to these operations. In 2010, Majumdar and Samanta [13] defined generalised fuzzy soft sets and studied some of their properties. They also gave applications of generalised fuzzy soft sets in decision making problem and medical diagnosis problem.

Also in 2010 Xiao et al. [14] proposed a combined forecasting approach based on fuzzy soft sets (CFFSS) by using an export dataset of Chongqing Municipality China from 1993 to 2006 and compares a CFFSS with the combined forecasting approach based on the rough sets theory (CFRS). This approach constructed the fuzzy membership function and the tabular form of the fuzzy soft sets model. Ça ğ man et al. in 2011 [15] introduced fuzzy parameterized (FP)-soft sets and their related properties and proposed a decision making method based on FP-soft set theory. Ça ğ man et al. in 2010 [16] defined fuzzy parameterized fuzzy soft sets (fpfs-sets) and their operations. They then presented the decision making method on the $f p f s$-set theory and provided an example that demonstrated that this method can work successfully. It can be applied to problems on many fields that contain uncertainty.

Alkhazaleh et al. [17] generalized the concept of fuzzy soft set to possibility fuzzy soft set and they gave some applications of this concept in decision making and medical diagnosis. They also introduced the concept of fuzzy parameterized interval-valued fuzzy soft set [18], where the mapping is defined from the fuzzy set parameters to the interval-valued fuzzy subsets of the universal set, and gave an application of this concept in decision making. Alkhazaleh and Salleh [19] introduced the concept of soft expert sets where the user can know the opinion of all experts in one model and also gave an application of this concept in decision-making problem. Alkhazaleh and Salleh [20] generalized the concept of a soft expert set to fuzzy soft expert set, which is a more effective and useful. They also defined its basic operations, namely complement, union, intersection, AND and OR, and gave an application of this concept in decision-making problem. They also studied a mapping on fuzzy soft expert classes and its properties. As a generalization of Molodtsov's soft set, Alkhazaleh et al. [21] presented the definition of a soft multiset and its basic operations such as complement, union and intersection. In 2012 Alkhazaleh and Salleh [22] introduced the concept of fuzzy soft multiset as a combination of soft multiset and fuzzy set and studied its properties and operations. They presented the applications of this concept to decisionmaking problems. In 2012 Salleh et al. [23] introduced the notion of multiparameterized soft set and studied its properties. Yang et al. [24] presented the concept of interval-valued fuzzy soft set by combining the intervalvalued fuzzy set [25] [26] and soft set models. In 2011 Sebastian and Ramakrishnan [27] proposed the concept of the multi-fuzzy set which is a more general fuzzy set using ordinary fuzzy sets as building blocks, its membership function is an ordered sequence of ordinary fuzzy membership functions. The notion of multi-fuzzy sets provides a new method to represent some problems which are difficult to explain in other extensions of fuzzy set theory, such as the color of pixels. Yang et al. [28] in 2012 introduced the concept of multi-fuzzy soft set which is a combination of multi-fuzzy set and soft set and studied and its basic operations such as complement, union and intersection. They also introduced the application of this concept in decision making. For more information on soft set and fuzzy soft set, see Abdul Razak Salleh 2011 [29]. In this paper, we introduce the concept of multi-interval-valued fuzzy soft set (M-IVFSS). We also define its basic operations, namely complement, union, intersection, AND and OR. Finally, we give an application of this concept in decision-making problem.

\section{Preliminaries}

In this section we recall some definitions and properties required in this paper.

Definition 1 [26] An interval-valued fuzzy set $\tilde{X}$ on a universe $U$ is a mapping such that

$$
\tilde{X}: U \rightarrow \operatorname{Int}([0,1])
$$


where $\operatorname{Int}([0,1])$ stands for the set of all closed subintervals of $[0,1]$, the set of all interval-valued fuzzy sets on $U$ is denoted by $\tilde{P}(U)$.

Suppose that $\tilde{X} \in \tilde{P}(U), \forall x \in U, \mu_{x}(x)=\left[\mu_{x}^{-}(x), \mu_{x}^{+}(x)\right]$ is called the degree of membership of an element $x$ to $X$. $\mu_{x}^{-}(x)$ and $\mu_{x}^{+}(x)$ are referred to as the lower and upper degrees of membership of $x$ to $X$ where $0 \leq \mu_{x}^{-}(x) \leq \mu_{x}^{+}(x) \leq 1$.

Definition 2 [25] The subset, complement, intersection and union of the interval-valued fuzzy sets are defined as follows: Let $\tilde{X}, \tilde{Y} \in \tilde{P}(U)$ then

1) The complement of $\tilde{X}$ is denoted by $\tilde{X}^{c}$ where

$$
\mu_{\tilde{X}^{c}}(x)=1-\mu_{\tilde{X}}(x)=\left[1-\mu_{\tilde{X}}^{+}(x), 1-\mu_{\tilde{X}}^{-}(x)\right] ;
$$

2) The intersection of $\tilde{X}$ and $\tilde{Y}$ is denoted by $\tilde{X} \cap \tilde{Y}$ where

$$
\mu_{\tilde{X} \cap \tilde{Y}}(x)=\inf \left[\mu_{\tilde{X}}(x), \mu_{\tilde{Y}}(x)\right]=\left[\inf \left(\mu_{\tilde{X}}^{-}(x), \mu_{\tilde{Y}}^{-}(x)\right), \inf \left(\mu_{\tilde{X}}^{+}(x), \mu_{\tilde{Y}}^{+}(x)\right)\right] ;
$$

3) The union of $\tilde{X}$ and $\tilde{Y}$ is denoted by $\tilde{X} \cup \tilde{Y}$ where

$$
\mu_{\tilde{X} \cup \tilde{Y}}(x)=\sup \left[\mu_{\tilde{X}}(x), \mu_{\tilde{Y}}(x)\right]=\left[\sup \left(\mu_{\tilde{X}}^{-}(x), \mu_{\tilde{Y}}^{-}(x)\right), \sup \left(\mu_{\tilde{X}}^{+}(x), \mu_{\tilde{Y}}^{+}(x)\right)\right] ;
$$

4) $X$ is a subset of $Y$ denoted by $X \subseteq Y$ if $\mu_{X}^{-}(x) \leq \mu_{Y}^{-}(x)$ and $\mu_{X}^{+}(x) \leq \mu_{Y}^{+}(x)$.

Molodtsov defined soft set in the following way. Let $U$ be a universe and $E$ be a set of parameters. Let $P(U)$ denote the power set of $U$ and $A \subseteq E$.

Definition 3 [1] A pair $(F, A)$ is called a soft set over $U$, where $F$ is a mapping

$$
F: A \rightarrow P(U) .
$$

In other words, a soft set over $U$ is a parameterized family of subsets of the universe $U$. For $\varepsilon \in A, F(\varepsilon)$ may be considered as the set of $\varepsilon$-approximate elements of the soft set $(F, A)$.

Definition 4 [5] Let $U$ be an initial universal set and let $E$ be a set of parameters. Let $I^{U}$ denote the power set of all fuzzy subsets of $U$. Let $A \subseteq E$. A pair $(F, E)$ is called a fuzzy soft set over $U$ where $F$ is a mapping given by

$$
F: A \rightarrow I^{U} \text {. }
$$

Definition 5 [24] Let $U$ be an initial universe and $E$ be a set of parameters. $\tilde{P}(U)$ denotes the set of all interval-valued fuzzy sets of $U$. Let $A \subseteq E$. A pair $(\tilde{F}, A)$ is an interval-valued fuzzy soft set over $U$, where $\tilde{F}$ is a mapping given by $\tilde{F}: A \rightarrow \tilde{P}(U)$.

Definition 6 [27] Let $k$ be a positive integer, a multi-fuzzy set $\tilde{A}$ in $U$ is a set of ordered sequences

$$
\tilde{A}=\left\{\frac{u}{\left(\mu_{1}(u), \mu_{2}(u), \cdots, \mu_{i}(u), \cdots, \mu_{k}(u)\right)}: u \in U\right\}
$$

where $\mu_{i}(u) \in \tilde{P}(U), i=1,2, \cdots, k$. The function $\mu_{\tilde{A}}=\left\{\mu_{1}, \mu_{2}, \cdots, \mu_{k}\right\}$ is called the multi-membership function of multi-fuzzy set $\tilde{A} ; k$ is called the dimension of $\tilde{A}$. The set of all multi-fuzzy sets of dimension $k$ in $U$ is denoted by $M^{k} F S(U)$.

Remark 7 [27] Clearly, a multi-fuzzy set of dimension 1 is a Zadeh fuzzy set, and a multi-fuzzy set of dimension 2 with $\mu_{1}(u)+\mu_{2}(u) \leq 1$ is an Atanassov intuitionistic fuzzy set.

Remark 8 [27] If $\sum_{i=1}^{k} \mu_{i}(u) \leq 1, \forall u \in U$, then the multi-fuzzy set of dimension $k$ is called a normalized multifuzzy set. If $\sum_{i=1}^{k} \mu_{i}(u)=l>1$, for some $u \in U$, we redefine the multi-membership degree $\left(\mu_{1}(u), \mu_{2}(u), \cdots, \mu_{k}(u)\right)$ as $\frac{1}{l}\left(\mu_{1}(u), \mu_{2}(u), \cdots, \mu_{k}(u)\right)$ then the non-normalized multi-fuzzy set can be changed into a normalized multi-fuzzy set. 
Definition 9 [27] Let $\tilde{A} \in M^{k} F S(U)$. If $\tilde{A}=\left\{\frac{u}{(0,0, \cdots, 0)}: u \in U\right\}$, then $\tilde{A}$ is called the null multi-fuzzy set of dimension $k$, denoted by $\tilde{\phi}_{k}$. If $\tilde{A}=\left\{\frac{u}{(1,1, \cdots, 1)}: u \in U\right\}$, then $\tilde{A}$ is called the absolute multi-fuzzy set of dimension $k$, denoted by $\tilde{1}_{k}$.

Definition 10 [27] Let $\tilde{A}=\left\{\frac{u}{\left(\mu_{1}(u), \mu_{2}(u), \cdots, \mu_{k}(u)\right)}: u \in U\right\}$ and $\tilde{B}=\left\{\frac{u}{\left(v_{1}(u), v_{2}(u), \cdots, v_{k}(u)\right)}: u \in U\right\}$ be two multi-fuzzy sets of dimension $k$ in $U$. We define the following relations and operations:

1) $\tilde{A} \sqsubseteq \tilde{B}$ iff $\mu_{i}(u) \leq v_{i}(u), \forall u \in U$ and $1 \geq i \leq k$.

2) $\tilde{A}=\tilde{B}$ iff $\mu_{i}(u)=v_{i}(u), \forall u \in U$ and $1 \geq i \leq k$.

3) $\tilde{A} \sqcup \tilde{B}=\left\{\frac{u}{\left(\mu_{1}(u) \vee v_{1}(u), \mu_{2}(u) \vee v_{2}(u), \cdots, \mu_{k}(u) \vee v_{k}(u)\right)}: u \in U\right\}$

4) $\tilde{A} \sqcap \tilde{B}=\left\{\frac{u}{\left(\mu_{1}(u) \wedge v_{1}(u), \mu_{2}(u) \wedge v_{2}(u), \cdots, \mu_{k}(u) \wedge v_{k}(u)\right)}: u \in U\right\}$

5) $\tilde{A}^{c}=\left\{\frac{u}{\left(\mu_{1}^{c}(u), \mu_{2}^{c}(u), \cdots, \mu_{k}^{c}(u)\right)}: u \in U\right\}$

Definition 11 [28] A pair $(\tilde{F}, A)$ is called a multi-fuzzy soft set of dimension $k$ over $U$, where $\tilde{F}$ is a mapping given by

$$
\tilde{F}: A \rightarrow M^{k} F S(U) .
$$

A multi-fuzzy soft set is a mapping from parameters to $M^{k} F S(U)$. It is a parameterized family of multifuzzy subsets of $U$. For $e \in A ; \tilde{F}(e)$ may be considered as the set of $e$-approximate elements of the multifuzzy soft set $(\tilde{F}, A)$.

Example 12 [28] Suppose that $U=\left\{c_{1} ; c_{2} ; c_{3} ; c_{4} ; c_{5}\right\}$ is the set of color cloths under consideration, $A=\left\{e_{1} ; e_{2} ; e_{3}\right\}$ is the set of parameters, where $e_{1}$ stands for the parameter? color? which consists of red, green and blue, $e_{2}$ stands for the parameter? ingredient? which is made from wool, cotton and acrylic, and $e_{3}$ stands for the parameter? price? which can be various: high, medium and low. We define a multi-fuzzy soft set of dimension 3 as follows:

$$
\begin{aligned}
& \tilde{F}\left(e_{1}\right)=\left\{\frac{c_{1}}{(0.4 ; 0.2 ; 0.3)} ; \frac{c_{2}}{(0.2 ; 0.1 ; 0.6)} ; \frac{c_{3}}{(0.1 ; 0.3 ; 0.4)} ; \frac{c_{4}}{(0.3 ; 0.1 ; 0.3)} ; \frac{c_{5}}{(0.7 ; 0.1 ; 0.2)}\right\} ; \\
& \tilde{F}\left(e_{2}\right)=\left\{\frac{c_{1}}{(0.1 ; 0.2 ; 0.6)} ; \frac{c_{2}}{(0.3 ; 0.2 ; 0.4)} ; \frac{c_{3}}{(0.5 ; 0.3 ; 0.1)} ; \frac{c_{4}}{(0.6 ; 0.1 ; 0.3)} ; \frac{c_{5}}{(0.6 ; 0.2 ; 0.1)}\right\} ; \\
& \tilde{F}\left(e_{3}\right)=\left\{\frac{c_{1}}{(0.3 ; 0.2 ; 0.1)} ; \frac{c_{2}}{(0.4 ; 0.1 ; 0.2)} ; \frac{c_{3}}{(0.2 ; 0.2 ; 0.5)} ; \frac{c_{4}}{(0.7 ; 0.1 ; 0.2)} ; \frac{c_{5}}{(0.5 ; 0.2 ; 0.3)}\right\} .
\end{aligned}
$$

Definition 13 [28] Let $A ; B \in E$. Let $(\tilde{F}, A)$ and $(\tilde{G}, B)$ be two multi-fuzzy soft sets of dimension $k$ over $U$. $(\tilde{F}, A)$ is said to be a multi-fuzzy soft subset of $(\tilde{G}, B)$ if

1) $A \subseteq B$;

2) $\forall e \in A, \tilde{F}(e) \sqsubseteq \tilde{G}(e)$

In this case, We write $(\tilde{F}, A) \sqsubseteq(\tilde{G}, B)$. 
Definition 14 [28] The complement of a multi-fuzzy soft set $(\tilde{F}, A)$ of dimension $k$ over $U$ is denoted by $(\tilde{F}, A)^{c}$ and is defined by

$$
(\tilde{F}, A)^{c}=\left(\tilde{F}^{c}, A\right)
$$

where $\tilde{F}^{c}: A \rightarrow M^{k} F S(U)$ is a mapping given by $\tilde{F}^{c}(e)=(\tilde{F}(e))^{c}, \forall e \in A$.

Definition 15 [28] If $(\tilde{F}, A)$ and $(\tilde{G}, B)$ are two multi-fuzzy soft sets of dimension $k$ over $U$ the " $(\tilde{F}, A)$ AND $(\tilde{G}, B)$ ", denoted by $(\tilde{F}, A) \wedge(\tilde{G}, B)=(\tilde{H}, A \times B)$, where $\tilde{H}(\alpha, \beta)=\tilde{F}(\alpha) \sqcap \tilde{G}(\beta), \quad \forall(\alpha, \beta) \in A \times B$.

Definition 16 [28] If $(\tilde{F}, A)$ and $(\tilde{G}, B)$ are two multi-fuzzy soft sets of dimension $k$ over $U$ the " $(\tilde{F}, A)$ OR $(\tilde{G}, B)$ ", denoted by $(\tilde{F}, A) \vee(\tilde{G}, B)=(\tilde{H}, A \times B)$, where $\tilde{H}(\alpha, \beta)=\tilde{F}(\alpha) \sqcup \tilde{G}(\beta), \forall(\alpha, \beta) \in A \times B$.

Definition 17 [28] Union of two multi-fuzzy soft sets $(\tilde{F}, A)$ and $(\tilde{G}, B)$ of dimension $k$ over $U$, is the multi-fuzzy soft set $(\tilde{H}, C)$ where $C=A \cup B$, and $\forall \varepsilon \in C$,

$$
\tilde{H}(\varepsilon)= \begin{cases}\tilde{F}(\varepsilon), & \text { if } \varepsilon \in A-B \\ \tilde{G}(\varepsilon), & \text { if } \varepsilon \in B-A \\ \tilde{F}(\varepsilon) \sqcup \tilde{G}(\varepsilon), & \text { if } \varepsilon \in A \bigcap B\end{cases}
$$

Definition 18 [28] Intersection of two multi-fuzzy soft sets $(\tilde{F}, A)$ and $(\tilde{G}, B)$ of dimension $k$ over $U$, is the multi-fuzzy soft set $(\tilde{H}, C)$ where $C=A \cup B$, and $\forall \varepsilon \in C$,

$$
\tilde{H}(\varepsilon)= \begin{cases}\tilde{F}(\varepsilon), & \text { if } \varepsilon \in A-B \\ \tilde{G}(\varepsilon), & \text { if } \varepsilon \in B-A \\ \tilde{F}(\varepsilon) \sqcap \tilde{G}(\varepsilon), & \text { if } \varepsilon \in A \cap B\end{cases}
$$

\section{Multi-Interval-Valued-Fuzzy Soft Sets}

In this section we introduce the concept of multi-interval-valued-fuzzy soft sets as generalisation of definition given by [28]. We also give basic properties of this concept.

Before we define the concept of multi-interval-valued-fuzzy soft sets, we define the concept of multi-intervalvalued-fuzzy sets as follows:

Definition 19 Let $k$ be a positive integer, a multi-intrval-valued fuzzy set $\tilde{A}$ in $U$ is a set of ordered sequences

$$
\tilde{A}=\left\{\frac{u}{\left(\mu_{1}(u), \mu_{2}(u), \cdots, \mu_{i}(u), \cdots, \mu_{k}(u)\right)}: u \in U\right\}
$$

where $\mu_{i}(u) \in \widetilde{\operatorname{Int}}(U), i=1,2, \cdots, k$. The function $\mu_{\tilde{A}}=\left\{\mu_{1}, \mu_{2}, \cdots, \mu_{k}\right\}$ is called the multi-membership function of multi-interval-valued-fuzzy set $\tilde{A} ; k$ is called the dimension of $\tilde{A}$. The set of all multi-intervalvalued-fuzzy sets of dimension $k$ in $U$ is denoted by $M^{k} I V S(U)$.

Definition 20 A pair $(\tilde{F}, A)$ is called a multi-interval-valued-fuzzy soft set of dimension k over $U$, where $\tilde{F}$ is a mapping given by

$$
\tilde{F}: A \rightarrow M^{k} \operatorname{Int}(U)
$$

A multi-interval-valued-fuzzy soft set is a mapping from parameters to $M^{k} \operatorname{Int}(U)$. It is a parameterized family of multi-interval-valued-fuzzy subsets of $U$. For $e \in A ; \tilde{F}(e)$ may be considered as the set of $e$-approximate elements of the multi-interval-valued fuzzy soft set $(\tilde{F}, A)$.

Example 21 Suppose that $U=\left\{c_{1} ; c_{2} ; c_{3}\right\}$ is the set of color cloths under consideration, $A=\left\{e_{1} ; e_{2} ; e_{3}\right\}$ is the set of parameters, where $e_{1}$ stands for the parameter "color" which consists of red, green and blue, $e_{2}$ stands for the parameter "ingredient" which is made from wool, cotton and acrylic, and $e_{3}$ stands for the parameter "price" which can be various: high, medium and low. We define a multi-interval-valued-fuzzy soft set of dimension 3 as follows: 


$$
\begin{gathered}
\tilde{F}\left(e_{1}\right)=\left\{\frac{c_{1}}{([0.4,0.6] ;[0.1,0.4] ;[0.4,0.5])} ; \frac{c_{2}}{([0.5,0.8] ;[0.4,0.6] ;[0,0.2])} ; \frac{c_{3}}{([0.1,0.3] ;[0.2,0.5] ;[0.5,0.6])}\right\} ; \\
\tilde{F}\left(e_{2}\right)=\left\{\frac{c_{1}}{([0.1,0.3] ;[0.3,0.4] ;[0.5,0.6])} ; \frac{c_{2}}{([0.2,0.5] ;[0.1,0.4] ;[0.3,0.6])} ; \frac{c_{3}}{([0.3,0.5] ;[0.1,0.2] ;[0,0.1])}\right\} ; \\
\tilde{F}\left(e_{3}\right)=\left\{\frac{c_{1}}{([0.6,0.9] ;[0.4,0.6] ;[0.7,0.8])} ; \frac{c_{3}}{([0.3,0.5] ;[0.1,0.5] ;[0,0.2])} ; \frac{c_{3}}{([0.3,0.7] ;[0.2,0.5] ;[0.4,0.7])}\right\} .
\end{gathered}
$$

Definition 22 Let $A ; B \in E$. Let $(\tilde{F}, A)$ and $(\tilde{G}, B)$ be two multi-interval-valued-fuzzy soft sets of dimension k over $U$. $(\tilde{F}, A)$ is said to be a multi-interval-valued-fuzzy soft subset of $(\tilde{G}, B)$ if

1) $A \subseteq B$

2) $\forall e \in A, \tilde{F}(e) \sqsubseteq \tilde{G}(e)$

In this case, We write $(\tilde{F}, A) \sqsubseteq(\tilde{G}, B)$.

Example 23 Consider Example 21 where $A=\left\{e_{1} ; e_{2}\right\}$ and $B=\left\{e_{1} ; e_{2} ; e_{3}\right\}$. Clearly, $A \subset B$. Suppose $(\tilde{F}, A)$ and $(\tilde{G}, B)$ are two multi-interval-valued-fuzzy soft sets of dimension 3 defined as follows

$$
\begin{aligned}
& \tilde{F}\left(e_{1}\right)=\left\{\frac{c_{1}}{([0.4,0.6] ;[0.1,0.4] ;[0.4,0.5])} ; \frac{c_{2}}{([0.5,0.8] ;[0.4,0.6] ;[0,0.2])} ; \frac{c_{3}}{([0.1,0.3] ;[0.2,0.5] ;[0.5,0.6])}\right\} ; \\
& \tilde{F}\left(e_{2}\right)=\left\{\frac{c_{1}}{([0.1,0.3] ;[0.3,0.4] ;[0.5,0.6])} ; \frac{c_{2}}{([0.2,0.5] ;[0.1,0.4] ;[0.3,0.6])} ; \frac{c_{3}}{([0.3,0.5] ;[0.1,0.2] ;[0,0.1])}\right\} ;
\end{aligned}
$$

and

$$
\begin{aligned}
& \tilde{G}\left(e_{1}\right)=\left\{\frac{c_{1}}{([0.5,0.7] ;[0.3,0.4] ;[0.5,0.8])} ; \frac{c_{2}}{([0.6,0.9] ;[0.5,0.9] ;[0.2,0.4])} ; \frac{c_{3}}{([0.4,0.5] ;[0.3,0.7] ;[0.6,0.8])}\right\} ; \\
& \tilde{G}\left(e_{2}\right)=\left\{\frac{c_{1}}{([0.3,0.5] ;[0.6,0.7] ;[0.5,0.7])} ; \frac{c_{2}}{([0.3,0.6] ;[0.2,0.5] ;[0.4,0.6])} ; \frac{c_{3}}{([0.3,0.5] ;[0.3,0.4] ;[0,0.1])}\right\} ; \\
& \tilde{G}\left(e_{3}\right)=\left\{\frac{c_{1}}{([0.6,1] ;[0.5,0.6] ;[0.7,0.9])} ; \frac{c_{2}}{([0.4,0.6] ;[0.3,0.5] ;[0.1,0.3])} ; \frac{c_{3}}{([0.5,0.7] ;[0.4,0.6] ;[0.5,0.8])}\right\} .
\end{aligned}
$$

It is clear that $(\tilde{F}, A)$ is multi-interval-valued-fuzzy soft subset of $(\tilde{G}, B)$.

Definition 24 The complement of a multi-interval-valued-fuzzy soft set $(\tilde{F}, A)$ of dimension $k$ over $U$ is denoted by $(\tilde{F}, A)^{c}$ and is defined by

$$
(\tilde{F}, A)^{c}=\left(\tilde{F}^{c}, A\right)
$$

where $\tilde{F}^{c}: A \rightarrow M^{k} \operatorname{Int}(U)$ is a mapping given by $\tilde{F}^{c}(e)=(\tilde{F}(e))^{c}, \forall e \in A$.

Example 25 Consider Example 21 where

$$
\begin{aligned}
& \tilde{F}\left(e_{1}\right)=\left\{\frac{c_{1}}{([0.4,0.6] ;[0.1,0.4] ;[0.4,0.5])} ; \frac{c_{2}}{([0.5,0.8] ;[0.4,0.6] ;[0,0.2])} ; \frac{c_{3}}{([0.1,0.3] ;[0.2,0.5] ;[0.5,0.6])}\right\} ; \\
& \tilde{F}\left(e_{2}\right)=\left\{\frac{c_{1}}{([0.1,0.3] ;[0.3,0.4] ;[0.5,0.6])} ; \frac{c_{2}}{([0.2,0.5] ;[0.1,0.4] ;[0.3,0.6])} ; \frac{c_{3}}{([0.3,0.5] ;[0.1,0.2] ;[0,0.1])}\right\} ;
\end{aligned}
$$




$$
\tilde{F}\left(e_{3}\right)=\left\{\frac{c_{1}}{([0.6,0.9] ;[0.4,0.6] ;[0.7,0.8])} ; \frac{c_{2}}{([0.3,0.5] ;[0.1,0.5] ;[0,0.2])} ; \frac{c_{3}}{([0.3,0.7] ;[0.2,0.5] ;[0.4,0.7])}\right\} .
$$

By using interval-valued fuzzy complement for $\tilde{F}(e)$ we have

$$
\begin{aligned}
\tilde{F}^{c}\left(e_{1}\right) & =\left\{\frac{c_{1}}{([0.4,0.6] ;[0.6,0.9] ;[0.5,0.6])} ; \frac{c_{2}}{([0.2,0.5] ;[0.4,0.6] ;[0.8,1])} ; \frac{c_{3}}{([0.7,0.9] ;[0.5,0.8] ;[0.4,0.5])}\right\} ; \\
\tilde{F}^{c}\left(e_{2}\right) & =\left\{\frac{c_{1}}{([0.7,0.9] ;[0.6,0.7] ;[0.4,0.5])} ; \frac{c_{2}}{([0.5,0.8] ;[0.6,0.9] ;[0.4,0.7])} ; \frac{c_{3}}{([0.5,0.7] ;[0.8,0.9] ;[0.9,1])}\right\} ; \\
\tilde{F}^{c}\left(e_{3}\right) & =\left\{\frac{c_{1}}{([0.1,0.4] ;[0.4,0.6] ;[0.2,0.3])} ; \frac{c_{2}}{([0.5,0.7] ;[0.5,0.9] ;[0.8,1])} ; \frac{c_{3}}{([0.3,0.7] ;[0.5,0.8] ;[0.3,0.6])}\right\} .
\end{aligned}
$$

Proposition 26 Let $(\tilde{F}, A)$ be a MIVFSS of dimension $k$ over $U$. Then the following holds:

$$
\left(\tilde{F}^{c}(e)\right)^{c}=\tilde{F}(e), \forall e \in A \text {. }
$$

Proof.

Let $\tilde{F}^{c}(e)=\tilde{G}(e)$ then

$$
\left(\tilde{F}^{c}(e)\right)^{c}=\tilde{G}^{c}(e)
$$

But from Definition $24 \tilde{G}(e)=\left(\tilde{F}^{c}(e)\right)$ then

$$
\tilde{G}(e)^{c}=\left(\tilde{F}^{c}(e)\right)^{c}=\tilde{F}(e)
$$

Definition 27 Union of two multi-interval-valued-fuzzy soft sets $(\tilde{F}, A)$ and $(\tilde{G}, B)$ of dimension $k$ over $U$, is the multi-interval-valued-fuzzy soft set $(\tilde{H}, C)$ where $C=A \cup B$, and $\forall \varepsilon \in C$,

$$
\tilde{H}(\varepsilon)= \begin{cases}\tilde{F}(\varepsilon), & \text { if } \varepsilon \in A-B \\ \tilde{G}(\varepsilon), & \text { if } \varepsilon \in B-A \\ \tilde{F}(\varepsilon) \cup \tilde{G}(\varepsilon), & \text { if } \varepsilon \in A \bigcap B\end{cases}
$$

Example 28 Consider Example 21 where $A=\left\{e_{1} ; e_{2} ; e_{3}\right\}$ and $B=\left\{e_{1} ; e_{2} ; e_{3}\right\}$. Suppose $(\tilde{F}, A)$ and $(\tilde{G}, B)$ are two multi-interval-valued-fuzzy soft sets of dimension 3 defined as follows

$$
\begin{aligned}
& \tilde{F}\left(e_{1}\right)=\left\{\frac{c_{1}}{([0.4,0.6] ;[0.1,0.4] ;[0.4,0.5])} ; \frac{c_{2}}{([0.5,0.8] ;[0.4,0.6] ;[0,0.2])} ; \frac{c_{3}}{([0.1,0.3] ;[0.2,0.5] ;[0.5,0.6])}\right\} ; \\
& \tilde{F}\left(e_{2}\right)=\left\{\frac{c_{1}}{([0.1,0.3] ;[0.3,0.4] ;[0.5,0.6])} ; \frac{c_{2}}{([0.2,0.5] ;[0.1,0.4] ;[0.3,0.6])} ; \frac{c_{3}}{([0.3,0.5] ;[0.1,0.2] ;[0,0.1])}\right\} ; \\
& \tilde{F}\left(e_{3}\right)=\left\{\frac{c_{1}}{([0.3,0.5] ;[0.7,0.8] ;[0.2,0.4])} ; \frac{c_{2}}{([0.3,0.4] ;[0.2,0.5] ;[0.3,0.6])} ; \frac{c_{3}}{([0.4,0.5] ;[0.4,0.6] ;[0.5,0.8])}\right\} ; \\
& \tilde{G}\left(e_{1}\right)=\left\{\frac{c_{1}}{([0.2,0.4] ;[0.1,0.3] ;[0.1,0.5])} ; \frac{c_{2}}{([0.4,0.5] ;[0.5,0.9] ;[0.2,0.4])} ; \frac{c_{3}}{([0.4,0.5] ;[0.3,0.7] ;[0.6,0.8])}\right\} ; \\
& \tilde{G}\left(e_{2}\right)=\left\{\frac{c_{1}}{([0.3,0.5] ;[0.6,0.7] ;[0.5,0.7])} ; \frac{c_{2}}{([0.3,0.6] ;[0.2,0.5] ;[0.5,0.7])} ; \frac{c_{3}}{([0.4,0.7] ;[0.1,0.3] ;[0,0.1])}\right\} ;
\end{aligned}
$$


$\tilde{G}\left(e_{3}\right)=\left\{\frac{c_{1}}{([0.4,0.6] ;[0.6,0.8] ;[0.8,0.9])} ; \frac{c_{2}}{([0.7,0.9] ;[0.3,0.5] ;[0.3,0.5])} ; \frac{c_{3}}{([0.2,0.4] ;[0.4,0.6] ;[0.3,0.5])}\right\} ;$

By using the interval-valued fuzzy union we have $(\tilde{F}, A) \cup(\tilde{G}, B)=(\tilde{H}, C)$, where

$\tilde{H}\left(e_{1}\right)=\left\{\frac{c_{1}}{([0.4,0.6] ;[0.1,0.4] ;[0.4,0.5])} ; \frac{c_{2}}{([0.5,0.8] ;[0.5,0.9] ;[0.2,0.4])} ; \frac{c_{3}}{([0.4,0.5] ;[0.3,0.7] ;[0.6,0.8])}\right\} ;$

$\tilde{H}\left(e_{2}\right)=\left\{\frac{c_{1}}{([0.3,0.5] ;[0.6,0.7] ;[0.5,0.7])} ; \frac{c_{2}}{([0.3,0.6] ;[0.2,0.5] ;[0.5,0.7])} ; \frac{c_{3}}{([0.4,0.7] ;[0.1,0.3] ;[0,0.1])}\right\} ;$

$\tilde{H}\left(e_{3}\right)=\left\{\frac{c_{1}}{([0.4,0.6] ;[0.7,0.8] ;[0.8,0.9])} ; \frac{c_{2}}{([0.7,0.9] ;[0.3,0.5] ;[0.3,0.6])} ; \frac{c_{3}}{([0.4,0.5] ;[0.4,0.7] ;[0.5,0.9])}\right\}$.

Proposition 29 Let $(\tilde{F}, A),(\tilde{G}, A)$ and $(\tilde{H}, A)$ be any three MIVFSSs of dimension $k$ over $U$. Then the following results hold:

1) $(\tilde{F}, A) \cup(\tilde{G}, A)=(\tilde{G}, A) \cup(\tilde{F}, A)$.

2) $(\tilde{F}, A) \cup((\tilde{G}, A) \cup(\tilde{H}, A))=((\tilde{F}, A) \cup(\tilde{G}, A)) \cup(\tilde{H}, A)$.

3) $(\tilde{F}, A) \cup(\tilde{F}, A)=(\tilde{F}, A)$.

Proof.

1) $(\tilde{F}, A) \cup(\tilde{G}, A)=(\tilde{R}, A)$

From Definition 27 and by consider the case when $\varepsilon \in B \cap C$ as the other cases are trivial, we have $\tilde{R}(e)=\tilde{F}(e) \cup \tilde{G}(e)=\tilde{G}(e) \cup \tilde{F}(e)$ (since the union of interval-valued fuzzy sets is commutative).

2) The proof is straightforward from Definition 27.

3) The proof is straightforward from Definition 27.

Definition 30 Intersection of two multi-interval-valued-fuzzy soft sets $(\tilde{F}, A)$ and $(\tilde{G}, B)$ of dimension $k$ over $U$, is the multi-interval-valued-fuzzy soft set $(\tilde{H}, C)$ where $C=A \cup B$, and $\forall \varepsilon \in C$,

$$
\tilde{H}(\varepsilon)= \begin{cases}\tilde{F}(\varepsilon), & \text { if } \varepsilon \in A-B \\ \tilde{G}(\varepsilon), & \text { if } \varepsilon \in B-A \\ \tilde{F}(\varepsilon) \cap \tilde{G}(\varepsilon), & \text { if } \varepsilon \in A \cap B\end{cases}
$$

Example 31 Consider Example 28. By using the interval-valued fuzzy intersection we have $(\tilde{F}, A) \cap(\tilde{G}, B)=(\tilde{H}, C)$, where

$$
\begin{gathered}
\tilde{H}\left(e_{1}\right)=\left\{\frac{c_{1}}{([0.2,0.4] ;[0.1,0.3] ;[0.1,0.5])} ; \frac{c_{2}}{([0.4,0.5] ;[0.4,0.6] ;[0,0.2])} ; \frac{c_{3}}{([0.1,0.3] ;[0.2,0.5] ;[0.5,0.6])}\right\} ; \\
\tilde{H}\left(e_{2}\right)=\left\{\frac{c_{1}}{([0.1,0.3] ;[0.3,0.4] ;[0.5,0.6])} ; \frac{c_{2}}{([0.2,0.5] ;[0.1,0.4] ;[0.3,0.6])} ; \frac{c_{3}}{([0.3,0.5] ;[0.1,0.2] ;[0,0.1])}\right\} ; \\
\tilde{H}\left(e_{3}\right)=\left\{\frac{c_{1}}{([0.3,0.5] ;[0.6,0.8] ;[0.2,0.4])} ; \frac{c_{2}}{([0.3,0.4] ;[0.2,0.5] ;[0.3,0.5])} ; \frac{c_{3}}{([0.2,0.4] ;[0.4,0.6] ;[0.3,0.5])}\right\} .
\end{gathered}
$$

Proposition 32 Let $(\tilde{F}, A),(\tilde{G}, A)$ and $(\tilde{H}, A)$ be any three MIVFSSs of dimension $k$ over $U$. Then the following results hold:

1) $(\tilde{F}, A) \cap(\tilde{G}, A)=(\tilde{G}, A) \cap(\tilde{F}, A)$.

2) $(\tilde{F}, A) \cap((\tilde{G}, A) \cap(\tilde{H}, A))=((\tilde{F}, A) \cap(\tilde{G}, A)) \cap(\tilde{H}, A)$. 
3) $(\tilde{F}, A) \cap(\tilde{F}, A)=(\tilde{F}, A)$.

Proof.

1) $(\tilde{F}, A) \cap(\tilde{G}, A)=(\tilde{R}, A)$

From Definition 30 and by consider the case when $\varepsilon \in B \cap C$ as the other cases are trivial, we have $\tilde{R}(e)=\tilde{F}(e) \cap \tilde{G}(e)=\tilde{G}(e) \cap \tilde{F}(e)$ (since intersection of interval-valued fuzzy sets is commutative).

2) The proof is straightforward from Definition 30.

3) The proof is straightforward from Definition 30.

Proposition 33 Let $(\tilde{F}, A)$ and $(\tilde{G}, A)$ be any two MIVFSSs of dimension $k$ over $U$. Then the DeMorgan's Laws hold:

1) $((\tilde{F}, A) \cup(\tilde{G}, A))^{c}=(\tilde{F}, A)^{c} \cap(\tilde{G}, A)^{c}$.

2) $((\tilde{F}, A) \cap(\tilde{G}, A))^{c}=(\tilde{F}, A)^{c} \cup(\tilde{G}, A)^{c}$.

Proof.

1) $(\tilde{F}, A)^{c} \cap(\tilde{G}, A)^{c}=\left(\tilde{F}^{c}(e) \cap \tilde{G}^{c}(e)\right)=((\tilde{F}(e)) \cup(\tilde{G}(e)))^{c}=((\tilde{F}, A) \cup(\tilde{G}, A))^{c}$.

2) The proof is similar to the above progress.

Proposition 34 Let $(\tilde{F}, A),(\tilde{G}, A)$ and $(\tilde{H}, A)$ be any three MIVFSSs of dimension $k$ over $U$. Then the following results hold:

1) $(\tilde{F}, A) \cup((\tilde{G}, A) \cap(\tilde{H}, A))=((\tilde{F}, A) \cup(\tilde{G}, A)) \cap((\tilde{F}, A) \cup(\tilde{H}, A))$.

2) $(\tilde{F}, A) \cap((\tilde{G}, A) \cup(\tilde{H}, A))=((\tilde{F}, A) \cap(\tilde{G}, A)) \tilde{U}((\tilde{F}, A) \cap(\tilde{H}, A))$.

Proof. a) For all $x \in E$,

$$
\begin{aligned}
& \lambda_{\tilde{F}(x) \cup(\tilde{G}(x) \cap \tilde{H}(x))}(x) \\
= & {\left[\sup _{i \rightarrow k}\left(\lambda_{\tilde{F}(x)}^{-}(x), \lambda_{(\tilde{G}(x) \cap \tilde{H}(x))}^{-}(x)\right), \sup _{i \rightarrow k}\left(\lambda_{\tilde{F}(x)}^{+}(x), \lambda_{(\tilde{G}(x) \cap \tilde{H}(x))}^{+}(x)\right)\right] } \\
= & {\left[\sup _{i \rightarrow k}\left(\lambda_{\tilde{F}(x)}^{-}(x), \inf _{i \rightarrow k}\left(\lambda_{\tilde{G}(x)}^{-}(x), \lambda_{\tilde{H}(x)}^{-}(x)\right)\right), \sup _{i \rightarrow k}\left(\lambda_{\tilde{F}(x)}^{+}(x), \inf _{i \rightarrow k}\left(\lambda_{\tilde{G}(x)}^{+}(x), \lambda_{\tilde{H}(x)}^{+}(x)\right)\right)\right] } \\
= & {\left[\inf _{i \rightarrow k}\left(\sup _{i \rightarrow k}\left(\lambda_{\tilde{F}(x)}^{-}(x), \lambda_{\tilde{G}(x)}^{-}(x)\right), \sup _{i \rightarrow k}\left(\lambda_{\tilde{F}(x)}^{-}(x), \lambda_{\tilde{H}(x)}^{-}(x)\right)\right),\right.} \\
& \left.\inf _{i \rightarrow k}\left(\sup _{i \rightarrow k}\left(\lambda_{\tilde{F}(x)}^{+}(x), \lambda_{\tilde{G}(x)}^{+}(x)\right), \sup _{i \rightarrow k}\left(\lambda_{\tilde{F}(x)}^{+}(x), \lambda_{\tilde{H}(x)}^{+}(x)\right)\right)\right] \\
= & \lambda_{\tilde{F}(x) \cup \tilde{G}(x)) \cap(\tilde{F}(x) \cup \tilde{H}(x))}(x)
\end{aligned}
$$

b) Similar to the proof of a.

Definition 35 If $(\tilde{F}, A)$ and $(\tilde{G}, B)$ are two multi-interval-valued-fuzzy soft sets of dimension $k$ over $U$ the “ $(\tilde{F}, A) \operatorname{AND}(\tilde{G}, B)$ ”, denoted by $(\tilde{F}, A) \wedge(\tilde{G}, B)=(\tilde{H}, A \times B)$, where $\tilde{H}(\alpha, \beta)=\tilde{F}(\alpha) \cap \tilde{G}(\beta)$, $\forall(\alpha, \beta) \in A \times B$.

Example 36 Consider Example 21. By using the interval-valued fuzzy union we have $(\tilde{F}, A)$ AND $(\tilde{G}, B)=(\tilde{H}, A \times B)$, where

$\tilde{H}\left(e_{1}, e_{1}\right)=\left\{\frac{c_{1}}{([0.4,0.6] ;[0.1,0.4] ;[0.4,0.5])} ; \frac{c_{2}}{([0.5,0.8] ;[0.5,0.9] ;[0.2,0.4])} ; \frac{c_{3}}{([0.4,0.5] ;[0.3,0.7] ;[0.6,0.8])}\right\} ;$
$\tilde{H}\left(e_{1}, e_{2}\right)=\left\{\frac{c_{1}}{([0.4,0.6] ;[0.6,0.7] ;[0.5,0.7])} ; \frac{c_{2}}{([0.5,0.8] ;[0.4,0.6] ;[0.5,0.7])} ; \frac{c_{3}}{([0.4,0.7] ;[0.2,0.5] ;[0.5,0.6])}\right\} ;$ 


$$
\begin{aligned}
& \tilde{H}\left(e_{1}, e_{3}\right)=\left\{\frac{c_{1}}{([0.4,0.6] ;[0.6,0.8] ;[0.8,0.9])} ; \frac{c_{2}}{([0.7,0.9] ;[0.4,0.6] ;[0.3,0.5])} ; \frac{c_{3}}{([0.2,0.4] ;[0.4,0.6] ;[0.5,0.6])}\right\} ; \\
& \tilde{H}\left(e_{2}, e_{1}\right)=\left\{\frac{c_{1}}{([0.2,0.4] ;[0.3,0.4] ;[0.5,0.6])} ; \frac{c_{2}}{([0.4,0.5] ;[0.5,0.9] ;[0.3,0.6])} ; \frac{c_{3}}{([0.4,0.5] ;[0.3,0.7] ;[0.6,0.8])}\right\} ; \\
& \tilde{H}\left(e_{2}, e_{2}\right)=\left\{\frac{c_{1}}{([0.3,0.5] ;[0.6,0.7] ;[0.5,0.7])} ; \frac{c_{2}}{([0.3,0.6] ;[0.2,0.5] ;[0.5,0.7])} ; \frac{c_{3}}{([0.4,0.7] ;[0.1,0.3] ;[0,0.1])}\right\} ; \\
& \tilde{H}\left(e_{2}, e_{3}\right)=\left\{\frac{c_{1}}{([0.4,0.6] ;[0.7,0.8] ;[0.8,0.9])} ; \frac{c_{2}}{([0.7,0.9] ;[0.3,0.5] ;[0.3,0.6])} ; \frac{c_{3}}{([0.3,0.5] ;[0.4,0.6] ;[0.3,0.5])}\right\} ; \\
& \tilde{H}\left(e_{3}, e_{1}\right)=\left\{\frac{c_{1}}{([0.3,0.5] ;[0.7,0.8] ;[0.2,0.5])} ; \frac{c_{2}}{([0.4,0.5] ;[0.5,0.9] ;[0.3,0.6])} ; \frac{c_{3}}{([0.4,0.5] ;[0.4,0.7] ;[0.6,0.8])}\right\} ; \\
& \tilde{H}\left(e_{3}, e_{2}\right)=\left\{\frac{c_{1}}{([0.3,0.5] ;[0.7,0.8] ;[0.5,0.7])} ; \frac{c_{2}}{([0.3,0.6] ;[0.2,0.5] ;[0.5,0.7])} ; \frac{c_{3}}{([0.4,0.7] ;[0.4,0.6] ;[0.5,0.8])}\right\} ; \\
& \tilde{H}\left(e_{3}, e_{3}\right)=\left\{\frac{c_{1}}{([0.4,0.6] ;[0.7,0.8] ;[0.8,0.9])} ; \frac{c_{2}}{([0.7,0.9] ;[0.3,0.5] ;[0.3,0.6])} ; \frac{c_{3}}{([0.4,0.5] ;[0.4,0.6] ;[0.5,0.8])}\right\} \text {. }
\end{aligned}
$$

Definition 37 If $(\tilde{F}, A)$ and $(\tilde{G}, B)$ are two multi-fuzzy soft sets of dimension $k$ over $U$ the " $(\tilde{F}, A)$ OR $(\tilde{G}, B)$ ", denoted by $(\tilde{F}, A) \vee(\tilde{G}, B)=(\tilde{H}, A \times B)$, where $\tilde{H}(\alpha, \beta)=\tilde{F}(\alpha) \cup \tilde{G}(\beta), \forall(\alpha, \beta) \in A \times B$.

Example 38 Consider Example 21. By using the interval-valued fuzzy intersection we have $(\tilde{F}, A)$ OR $(\tilde{G}, B)=(\tilde{H}, A \times B)$, where

$$
\begin{aligned}
& \tilde{H}\left(e_{1}, e_{1}\right)=\left\{\frac{c_{1}}{([0.2,0.4] ;[0.1,0.3] ;[0.1,0.5])} ; \frac{c_{2}}{([0.4,0.5] ;[0.4,0.6] ;[0.0 .2])} ; \frac{c_{3}}{([0.1,0.3] ;[0.2,0.5] ;[0.5,0.6])}\right\} ; \\
& \tilde{H}\left(e_{1}, e_{2}\right)=\left\{\frac{c_{1}}{([0.3,0.5] ;[0.1,0.4] ;[0.4,0.5])} ; \frac{c_{2}}{([0.3,0.6] ;[0.2,0.5] ;[0,0.2])} ; \frac{c_{3}}{([0.1,0.3] ;[0.1,0.3] ;[0,0.1])}\right\} ; \\
& \tilde{H}\left(e_{1}, e_{3}\right)=\left\{\frac{c_{1}}{([0.4,0.6] ;[0.1,0.4] ;[0.4,0.5])} ; \frac{c_{2}}{([0.5,0.8] ;[0.3,0.5] ;[0,0.2])} ; \frac{c_{3}}{([0.1,0.3] ;[0.2,0.5] ;[0.3,0.5])}\right\} \text {; } \\
& \tilde{H}\left(e_{2}, e_{1}\right)=\left\{\frac{c_{1}}{([0.1,0.3] ;[0.1,0.3] ;[0.1,0.5])} ; \frac{c_{2}}{([0.2,0.5] ;[0.1,0.4] ;[0.2,0.4])} ; \frac{c_{3}}{([0.3,0.5] ;[0.1,0.2] ;[0,0.1])}\right\} ; \\
& \tilde{H}\left(e_{2}, e_{2}\right)=\left\{\frac{c_{1}}{([0.1,0.3] ;[0.3,0.4] ;[0.5,0.6])} ; \frac{c_{2}}{([0.2,0.5] ;[0.1,0.4] ;[0.3,0.6])} ; \frac{c_{3}}{([0.3,0.5] ;[0.1,0.2] ;[0,0.1])}\right\} ; \\
& \tilde{H}\left(e_{2}, e_{3}\right)=\left\{\frac{c_{1}}{([0.1,0.3] ;[0.3,0.4] ;[0.5,0.6])} ; \frac{c_{2}}{([0.2,0.5] ;[0.1,0.4] ;[0.3,0.5])} ; \frac{c_{3}}{([0.2,0.4] ;[0.1,0.2] ;[0,0.1])}\right\} ; \\
& \tilde{H}\left(e_{3}, e_{1}\right)=\left\{\frac{c_{1}}{([0.2,0.4] ;[0.1,0.3] ;[0.1,0.4])} ; \frac{c_{2}}{([0.3,0.4] ;[0.2,0.5] ;[0.2,0.4])} ; \frac{c_{3}}{([0.4,0.5] ;[0.3,0.6] ;[0.5,0.8])}\right\} ;
\end{aligned}
$$




$$
\begin{gathered}
\tilde{H}\left(e_{3}, e_{2}\right)=\left\{\frac{c_{1}}{([0.3,0.5] ;[0.6,0.7] ;[0.2,0.4])} ; \frac{c_{2}}{([0.3,0.4] ;[0.2,0.5] ;[0.3,0.6])} ; \frac{c_{3}}{([0.4,0.5] ;[0.1,0.2] ;[0,0.1])}\right\} ; \\
\tilde{H}\left(e_{3}, e_{3}\right)=\left\{\frac{c_{1}}{([0.4,0.6] ;[0.6,0.8] ;[0.2,0.4])} ; \frac{c_{2}}{([0.3,0.4] ;[0.2,0.4] ;[0.2,0.5])} ; \frac{c_{3}}{([0.2,0.4] ;[0.4,0.6] ;[0.3,0.5])}\right\} .
\end{gathered}
$$

Proposition 39 Let $(\tilde{F}, A)$ and $(\tilde{G}, A)$ are two MIVFSSs of dimension $k$ over $U$. Then the following results hold:
1) $((\tilde{F}, A) \wedge(\tilde{G}, A))^{c}=(\tilde{F}, A)^{c} \vee(\tilde{G}, A)^{c}$,
2) $((\tilde{F}, A) \vee(\tilde{G}, A))^{c}=(\tilde{F}, A)^{c} \wedge(\tilde{G}, A)^{c}$,

Proof.

a) Suppose that $(\tilde{F}, A) \wedge(\tilde{G}, B)=(\tilde{O}, A \times B)$.

Therefore, $((\tilde{F}, A) \wedge(\tilde{G}, B))^{c}=(\tilde{O}, A \times B)^{c}=\left(\left(\tilde{O}^{c}(\alpha, \beta)\right),(A \times B)\right)$. Now,

$$
((\tilde{F}, A) \vee(\tilde{G}, B))^{c}=\left(\left(\left(\tilde{F}^{c}(\alpha)\right), A\right) \wedge\left(\left(\tilde{G}^{c}(\alpha)\right), B\right)\right)=(\tilde{J},(A \times B)),
$$

where $\tilde{J}(x, y)=\left(F^{c}(x)\right) \cap\left(G^{c}(x)\right)$.

Now, take $(\alpha, \beta) \in(A \times B)$.

Therefore,

$$
\begin{aligned}
(\tilde{O})^{c}(\alpha, \beta) & =\left(\tilde{c}\left[\inf _{i \rightarrow k}\left(\tilde{F}^{-}(\alpha), \tilde{G}^{-}(\beta)\right), \inf _{i \rightarrow k}\left(\tilde{F}^{+}(\alpha), \tilde{G}^{+}(\beta)\right)\right]\right) \\
& =\left(\left[1-\inf _{i \rightarrow k}\left(\tilde{F}^{+}(\alpha), \tilde{G}^{+}(\beta)\right), 1-\inf _{i \rightarrow k}\left(\tilde{F}^{+}(\alpha), \tilde{G}^{+}(\beta)\right)\right]\right) \\
& =\left(\left[\sup _{i \rightarrow k}\left(1-\tilde{F}^{+}(\alpha), 1-\tilde{G}^{+}(\beta)\right), \sup _{i \rightarrow k}\left(1-\tilde{F}^{+}(\alpha), 1-\tilde{G}^{+}(\beta)\right)\right]\right) \\
& =[(\tilde{c}(\tilde{F}(\alpha)) \tilde{U} \tilde{c}(\tilde{G}(\beta)))]=\tilde{J}(\alpha, \beta)
\end{aligned}
$$

Then $\tilde{O}^{c}$ and $\tilde{J}$ are the same. Hence, proved.

b) Similar to the proof of a.

\section{4. mivfs-Aggregation Operator}

In this section, we define an aggregate interval-valued fuzzy set of an MIVFS-set. We also define MIVFSaggregation operator that produces an aggregate interval-valued fuzzy set from an MIVFS-set and its parameter set. Also we give an application of this operator in decision making problem.

Definition 40 Let $(\tilde{F}, A) \in M I V F S S(U)$. Then a MIVFS-aggregation operator, denoted by $M_{I V F S}{ }_{a g g}$, is defined by

$$
\begin{gathered}
\operatorname{MIVFS}_{\text {agg }}: \tilde{F}(E) \times \operatorname{MIVFSS}(U) \rightarrow \operatorname{Int}(U), \\
\operatorname{MIVFS}_{\text {agg }}(\tilde{F}, A)=(\tilde{F}, A)^{*}
\end{gathered}
$$

where

$$
(\tilde{F}, A)^{*}=\left\{u / \mu_{(\tilde{F}, A)^{*}}(u): u \in U\right\}
$$

Is an interval-valued fuzzy set over $U$. The value $(\tilde{F}, A)^{*}$ is called an aggregate interval-valued fuzzy set of $(\tilde{F}, A)$. Here, the membership degree $\mu_{(\tilde{F}, A)^{*}}(u)$ of $u$ is defined as follows: 


$$
\mu_{(\tilde{F}, A)^{*}}(u)=\left[c^{-}=\frac{1}{k|A|} \sum_{x \in A 1 \rightarrow k} \mu_{\tilde{F}_{k}(x)^{-}}(u), c^{+}=\frac{1}{k|A|} \sum_{x \in A 1 \rightarrow k} \mu_{\tilde{F}_{k}(x)^{+}}(u)\right],
$$

where $|A|$ is the cardinality of $A$.

In the following example, we present an application of MIVFS-aggregation operator to solve a decision making problem.

\section{Example 41}

Step 1 Let the constructed MIVFS-set, $(\tilde{F}, A)$, be given as follows:

$$
\begin{aligned}
& \tilde{F}\left(e_{1}\right)=\left\{\frac{u_{1}}{([0.4,0.6] ;[0.1,0.4] ;[0.4,0.5])} ; \frac{u_{2}}{([0.5,0.8] ;[0.4,0.6] ;[0,0.2])} ; \frac{u_{3}}{([0.1,0.3] ;[0.2,0.5] ;[0.5,0.6])}\right\} ; \\
& \tilde{F}\left(e_{2}\right)=\left\{\frac{u_{1}}{([0.1,0.3] ;[0.3,0.4] ;[0.5,0.6])} ; \frac{u_{2}}{([0.2,0.5] ;[0.1,0.4] ;[0.3,0.6])} ; \frac{u_{3}}{([0.3,0.5] ;[0.1,0.2] ;[0,0.1])}\right\} ; \\
& \tilde{F}\left(e_{3}\right)=\left\{\frac{u_{1}}{([0.6,0.9] ;[0.4,0.6] ;[0.7,0.8])} ; \frac{u_{2}}{([0.3,0.5] ;[0.1,0.5] ;[0,0.2])} ; \frac{u_{3}}{([0.3,0.7] ;[0.2,0.5] ;[0.4,0.7])}\right\} .
\end{aligned}
$$

Step 2 The aggregate interval-valued fuzzy set can be found as

$$
(\tilde{F}, A)^{*}=\left\{\frac{u_{1}}{[0.388,0.566]}, \frac{u_{2}}{[0.211,0.477]}, \frac{u_{3}}{[0.233,0.455]}\right\}
$$

Step $3 \forall u_{i} \in U$, compute the score $r_{i}$ of $u_{i}$ such that

$$
r_{i}=\sum_{u_{j} \in U}\left(\left(c_{i}^{-}-c_{j}^{-}\right)+\left(c_{i}^{+}-c_{j}^{+}\right)\right)
$$

Thus, we have

$$
r_{1}=0.537, r_{2}=-0.266, r_{3}=-0.266
$$

Step 4 The decision is any one of the elements in $S$ where $S=\max _{u_{i} \in U}\left\{r_{i}\right\}$. In our example, $u_{1}$ is the best choice because $\max _{u_{i} \in U}\left\{r_{i}\right\}=r_{1}$.

\section{Conclusion}

As a generalisation of multi-fuzzy soft set and by combining this concept and interval-value fuzzy set, the concept of the multi-interval-valued fuzzy soft set is introduced and some of its properties studied. The complement, union and intersection, operations have been defined on the multi-interval-valued fuzzy soft set. An application of this theory is given in solving a decision making problem. We hope that our work would help enhancing this study on multi-fuzzy soft sets for the researchers.

\section{Acknowledgements}

We thank the Editor and the referee for their comments. Research of S. Alkhazaleh is funded by Shaqra University, Saudi Arabia. This support is greatly appreciated.

\section{References}

[1] Molodtsov, D. (1999) Soft Set Theory-First Results. Computers \& Mathematics with Applications, 37, $19-31$. http://dx.doi.org/10.1016/S0898-1221(99)00056-5

[2] Chen, D., Tsang, E.C.C., Yeung, D.S. and Wang, X. (2005) The Parameterization Reduction of Soft Sets and Its Application. Computers \& Mathematics with Applications, 49, 757-763. http://dx.doi.org/10.1016/j.camwa.2004.10.036

[3] Maji, P.K., Roy, A.R. and Biswas, R. (2003) Soft Set Theory. Computers \& Mathematics with Applications, 54, 555562. 
[4] Maji, P.K., Roy, A.R. and Biswas, R. (2002) An Application of Soft Sets in a Decision Making Problem. Computers \& Mathematics with Applications, 44, 1077-1083.

[5] Maji, P.K., Roy, A.R. and Biswas, R. (2001) Fuzzy Soft Sets. Journal of Fuzzy Mathematics, 9, 589-602.

[6] Roy, R. and Maji, P.K. (2007) A Fuzzy Soft Set Theoretic Approach to Decision Making Problems. Journal of Computational and Applied Mathematics, 203, 412-418. http://dx.doi.org/10.1016/j.cam.2006.04.008

[7] Ahmad, B. and Kharal, A. (2009) On Fuzzy Soft Sets. Advances in Fuzzy Systems, 6 p.

[8] Feng, F., Jun, Y.B., Liu, X. and Li, L. (2010) An Adjustable Approach to Fuzzy Soft Set Based Decision Making. Journal of Computational and Applied Mathematics, 234, 10-20. http://dx.doi.org/10.1016/j.cam.2009.11.055

[9] Yao, B.X., Liu, J. and Yan, R.X. (2008) Fuzzy Soft Set and Soft Fuzzy Set. IEEE Fourth International Conference on Natural Computation, 252-255.

[10] Kharal, A. and Ahmad, B. (2009) Mappings on Fuzzy Soft Classes. Advances in Fuzzy Systems, 6 p.

[11] Chaudhuri, A. and De, K. and Chatterjee, D. (2009) Solution of the Decision Making Problems Using Fuzzy Soft Relations. International Journal of Information Technology, 15, 78-107.

[12] Jiang, Y., Tang, Y., Chen, Q., Liu, H. and Tang, J. (2011) Extending Fuzzy Soft Sets with Fuzzy Description Logics. Knowledge-Based Systems, 24, 1096-1107. http://dx.doi.org/10.1016/j.knosys.2011.05.003

[13] Majumdar, P. and Samanta, S.K. (2010) Generalised Fuzzy Soft Sets. Computers and Mathematics with Applications, 59, 1425-1432. http://dx.doi.org/10.1016/j.camwa.2009.12.006

[14] Xiao, Z., Gong, K. and Zou, Y. (2010) A Combined Forecasting Approach Based on Fuzzy Soft Sets. Journal of Computational and Applied Mathematics, 228, 326-333. http://dx.doi.org/10.1016/j.cam.2008.09.033

[15] Çağman, N., Çitak, F. and Enginoglu, S. (2011) Fuzzy Parameterized Soft Set Theory and Its Applications. Annals of Fuzzy Mathematics and Informatics, 2, 219-226.

[16] Çağman, N., Çitak, F. and Enginoglu, S. (2010) Fuzzy Parameterized Fuzzy Soft Set Theory and Its Applications. Turkish Journal of Fuzzy Systems, 1, 21-35.

[17] Alkhazaleh, S., Salleh, A.R. and Hassan, N. (2011) Possibility Fuzzy Soft Set. Advances in Decision Sciences, 2011, Article ID: 479756.

[18] Alkhazaleh, S., Salleh, A.R. and Hassan, N. (2011) Fuzzy Parameterized Interval-Valued Fuzzy Soft Set. Applied Mathematical Sciences, 5, 3335-3346.

[19] Alkhazaleh, S. and Salleh, A.R. (2011) Soft Expert Sets. Advances in Decision Sciences, 2011, Article ID: 757868.

[20] Alkhazaleh, S. and Salleh, A.R. (2014) Fuzzy Soft Expert Set and Its Application. Applied Mathematics, 5, $1349-1368$. http://dx.doi.org/10.4236/am.2014.59127

[21] Alkhazaleh, S., Salleh, A.R. and Hassan, N. (2011) Soft Multisets Theory. Applied Mathematical Sciences, 5, 35613573.

[22] Alkhazaleh, S. and Salleh, A.R. (2012) Fuzzy Soft Multiset Theory. Abstract and Applied Analysis, 2012, Article ID: 350603.

[23] A.R. Salleh, Alkhazaleh, S., Hassan, N. and Ahmad, A.G. (2012) Multiparameterized Soft Set. Journal of Mathematics and Statistics, 8, 92-97. http://dx.doi.org/10.3844/jmssp.2012.92.97

[24] Yang, X.B., Lin, T.Y., Yang, J.Y., Li, Y. and Yu, D. (2009) Combination of Interval-Valued Fuzzy Set and Soft Set. Computers and Mathematics with Applications, 58, 521-527. http://dx.doi.org/10.1016/j.camwa.2009.04.019

[25] Gorzalczany, M.B. (1987) A Method of Inference in Approximate Reasoning Based on Interval Valued Fuzzy Sets. Fuzzy Sets and Systems, 21, 1-17. http://dx.doi.org/10.1016/0165-0114(87)90148-5

[26] Zadeh, L.A. (1975) The Concept of a Linguistic Variable and Its Application to Approximate Reasoning-I. Information Sciences, 8, 199-249. http://dx.doi.org/10.1016/0020-0255(75)90036-5

[27] Sebastian, S. and Ramakrishnan, T.V. (2011) Multi-Fuzzy Sets: An Extension of Fuzzy Sets. Fuzzy Information and Engineering, 1, 35-43.

[28] Yang, Y., Tan, X. and Meng, C. (2012) The Multi-Fuzzy Soft Set and Its Application in Decision Making. Applied Mathematical Modelling, 37, 4915-4923.

[29] Abdul Razak Salleh (2011) From Soft Sets to Intuitionistic Fuzzy Soft Sets: A Brief Survey. Proceedings of International Seminar on the Current Research Progress in Sciences and Technology (ISSTech 2011), Universiti Kebangsaan Malaysia-Universitas Indonesia, Bandung, Indonesia, October 2011. 